This document is the accepted manuscript version of the following article:

Tuzson, B., Jágerská, J., Looser, H., Graf, M., Fe1der, F., Fi11, M., ... Emmenegger, L. (2017). High1y selective volatile organic compounds breath analysis using a broadly-tunable vertical-externa1-cavity surface-emitting 1aser. Ana7ytical Chemistry. http://doi.org/10.1021/acs.analchem.6b04511

\title{
Highly Selective Volatile Organic Compounds Breath Analysis Using a Broadly-Tunable Vertical-External-Cavity Surface Emitting Laser
}

\author{
Béla Tuzson, ${ }^{*, \dagger}$ Jana Jágerská, ${ }^{\dagger, \dagger}$ Herbert Looser, ${ }^{\circledR}$ Manuel Graf, ${ }^{\dagger}$ Ferdinand \\ Felder, ${ }^{\S}$ Matthias Fill, ${ }^{\S}$ Luc Tappy, and Lukas Emmenegger ${ }^{\dagger}$ \\ $\dagger$ Laboratory for Air Pollution and Environmental Technology, Empa, 8600 Dübendorf, \\ Switzerland \\ $\ddagger$ Department of Physics and Technology, UiT - The Arctic University of Norway, 9019 \\ Tromsø, Norway \\ IInstitute for Aerosol and Sensor Technology, FHNW, 5210 Windisch, Switzerland \\ $\S$ Camlin Technologies AG, 8005 Zürich, Switzerland \\ \|Faculty of Biology and Medicine, Department of Physiology, UNIL, 1005 Lausanne, \\ Switzerland \\ E-mail: bela.tuzson@empa.ch
}

\section{Abstract}

A broadly tunable mid-infrared verticalexternal-cavity surface emitting laser (VECSEL) is employed in a direct absorption laser spectroscopic setup to measure breath acetone. The large wavelength coverage of more than $30 \mathrm{~cm}^{-1}$ at $3.38 \mu \mathrm{m}$ allows, in addition to acetone, the simultaneous measurement of isoprene, ethanol, methanol, methane, and water. Despite the severe spectral interferences from water and alcohols, an unambiguous determination of acetone is demonstrated with a precision of $13 \mathrm{ppbv}$ that is achieved after $5 \mathrm{~min}$ averaging at typical breath mean acetone levels in synthetic gas samples mimicking human breath.

\section{Introduction}

A very large number of volatile organic compounds (VOCs), produced from both endogenous and exogenous sources, appear at the trace level in human breath. Their elevated level or even the appearance of further compounds can provide valuable information about the physiological status of the body. Thus, breath analysis can be used as a noninvasive method for clinical diagnosis and therapeutic monitoring. ${ }^{1-3}$ Clearly, the development of analytical techniques that allow reliable and accurate quantification of volatile compounds in breath is of great interest. The major challenge is, however, to fulfil the requirements posed by clinical research, i.e. the analytical methods should allow on-line, realtime analysis, and simultaneous quantification of several metabolites over a wide concentration range in single breath exhalations. Furthermore, a high sample rate and hence rapid time response for analysis would be beneficial to assist rapid diagnosis. Consequently, a wide range of powerful analytical techniques has been developed for breath analysis. ${ }^{4}$ While sub-ppbv detection limit and on-line measurement capabilities were already demonstrated 
for a variety of compounds, the involved, mostly mass spectrometric based, instruments such as gas chromatography/mass spectrometry (GC$\mathrm{MS}),{ }^{5}$ proton-transfer-reaction-mass spectrometry (PTR-MS), ${ }^{6}$ and selected-ion flow-tube mass spectromety (SIFT-MS), ${ }^{7}$ are relatively large, complex, and expensive. These factors seriously hinder their broad application in medical cabinets and thus the routine exploitation of breath analysis for monitoring and therapeutic purposes.

In this paper, we propose an instrument based on tunable mid-infrared (mid-IR) laser absorption spectroscopy for selective and sensitive detection of acetone in a breath-like gas matrix. In particular, we have investigated the ability of this technique for on-line, real-time, and accurate quantification of acetone in the presence of the main interfering species like water, methane, and alcohols. The technology is suited for the development of a compact and mobile sensing platform to be deployed in a medical cabinet on a daily/hourly basis or even continuously.

The targeted application is to investigate whether breath acetone can be used as a biomarker for monitoring the ketotic state of an individual. This is motivated by the fact that being a byproduct of lipolysis/ketogenesis, acetone concentration increase correlates with rates of fat loss resulting either from fasting or from prolonged physical activity. ${ }^{8}$ Since hepatic ketogenesis closely parallels whole-body lipid oxidation, ${ }^{9}$ acetone has been suggested to provide real-time information on the effect of the lifestyle interventions targeted to stimulate fat oxidation. ${ }^{10}$ Isoprene, originating primarily from cholesterol biosynthesis, ${ }^{11}$ is an additional diagnostic parameter in the case of lipid metabolism disorders such as hypercholesterolemia, which is an established risk factor for coronary heart disease.

Laser spectroscopy is an established measurement technique for trace gas measurements, however, it's application in the field of breath research has been limited to just a few anorganic, simple molecular species, such as NO, CO, OCS, $\mathrm{NH}_{3}$, and $\mathrm{C}_{2} \mathrm{H}_{6} \cdot{ }^{12}$ This is mainly due to the fact that larger molecules like
VOC's exhibit broad and congested absorption spectra. The unambiguous detection, therefore, requires a wide tuning capabilities of the laser source. The most common distributed feedback (DFB) solutions, such as quantum cascade $^{13}$ (QC) and interband cascade ${ }^{14}$ (IC) lasers are, however, tunable only within a very narrow spectral range (typically $1 \mathrm{~cm}^{-1}$ ). Even though, the bound-to-continuum QCL design provides inherently broad gain devices, they require a wavelength selector such as a diffraction grating within an external cavity. ${ }^{15}$ This adds complexity, costs, and in case of external mechanical system, it reduces the tuning speed. A further challenge for external cavity QCLs is to address the $3 \mu \mathrm{m}$ spectral region. ${ }^{16}$ To overcome these limitations, we developed an instrument using an optically pumped, broadly tunable mid-infrared vertical-externalcavity surface-emitting laser (VECSEL). ${ }^{17,18}$

\section{Experimental}

\section{Spectral range selection}

The measurement technique exploits the alkene functional group absorption region of VOCs. The $\mathrm{C}-\mathrm{H}$ bond stretching vibration mode near $3.38 \mu \mathrm{m}$ has been selected based on the following considerations: i) both acetone and isoprene have overlapping absorption features with distinctive structures within the tuning range of the laser source, ii) the interferences from water and carbon-dioxide are low compared to other regions, and iii) the sharp absorption lines of $\mathrm{CH}_{4}$ can be used as precise frequency reference.

Figure 1 gives the spectral overview of the targeted metabolites in the mid-IR range. The simulation of VOCs is based on values from the Pacific Northwest National Laboratory (PNNL) database, ${ }^{19}$ while the absorption spectra of simple molecules such as water, carbon-dioxide and methane were calculated using the parameters from the HITRAN database ${ }^{20}$. For a realistic spectral simulation of the breath air matrix, typical average levels of the various species were assumed. Levels of isoprene have been reported at $\sim 200 \mathrm{ppbv}$, while those of ace- 

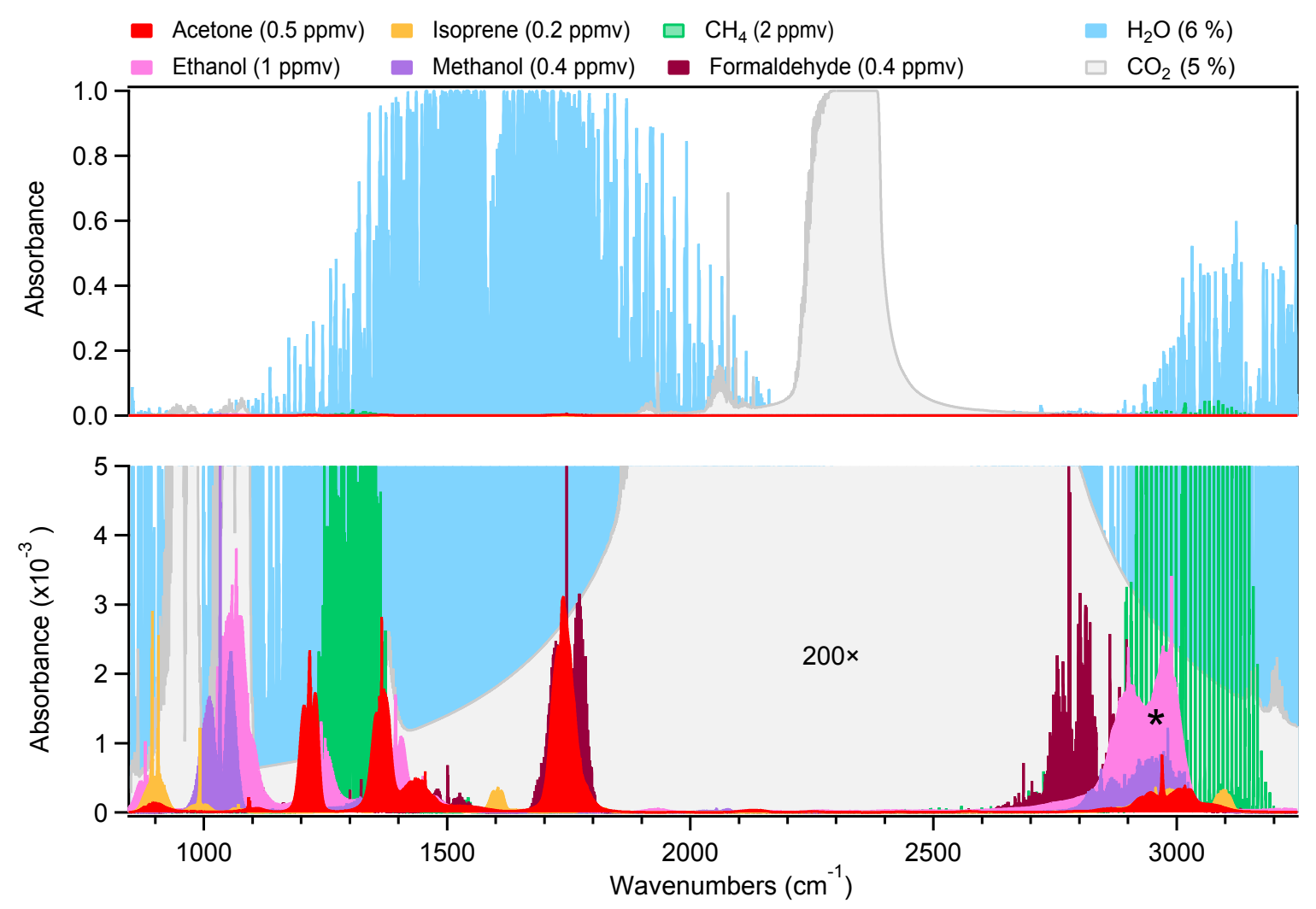

Figure 1: Overview of mid-IR absorption spectra of the most abundant species scaled by their typical mixing ratios as found in human breath. The simulations are based on HITRAN and PNNL data assuming $36 \mathrm{~m}$ optical path length and a gas pressure of $300 \mathrm{hPa}$. The lower graph scale is $200 \times$ magnified to highlight the absorption features of VOCs. The asterisk $(*)$ around $2970 \mathrm{~cm}^{-1}$ indicates the spectral range selected for simultaneous acetone and isoprene analysis.

tone can reach up to a few ppmv, depending upon the condition of the subject. ${ }^{5,21-23}$ Under these experimental conditions, the absorbance of VOCs is in the sub-\% range, while those of water and carbon-dioxide are fully dominating. These spectral conditions make VOC sensing challenging and, therefore, the various spectroscopic approaches developed up to date to determine acetone in human breath require almost exclusively a water removal step or even selective pre-concentration of the analyte (e.g. via cryogenic traps or chemical adsorbents) prior to analysis. ${ }^{24-27}$ The challenge of measuring VOCs in human breath directly, i.e. without any sample preparation, is well illustrated on the bottom graph of Figure 1. This close-up view further reveals a number of interferences from other compounds, mainly from alcohols (methanol, ethanol) and formaldehyde. Even for the most promising spectral windows, overlapping of spectral bands from different compounds may lead to spectral interferences. This demonstrates the necessity of broad-band multi-compound detection. Our choice of spectral window, also proposed by Arslanov et al. ${ }^{28}$, is located around $2970 \mathrm{~cm}^{-1}$, where already a modest tuneability of $30 \mathrm{~cm}^{-1}$ covers a range that fulfills the selection criteria listed above.

\section{Spectrometer design}

The mid-infrared light source is a VECSEL (Camlin Technologies AG, Switzerland), which consists of a Bragg mirror, a IV-VI semiconductors (lead chalcogenides) based active region (gain region) with several quantum wells (PbSe) in PbSrSe host material, and an external top mirror, which is movable in the vertical direction by a piezoelectric actuator. ${ }^{18}$ Due to the short cavity length of typically 


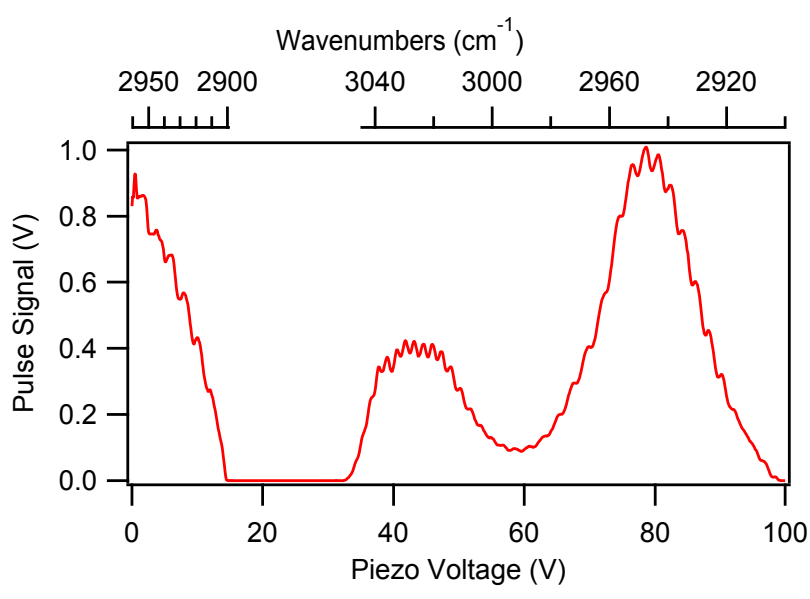

Figure 2: Tuning characteristics of the VECSEL as a function of the piezo voltage.

$25 \mu \mathrm{m}$, the emission occurs in a single longitudinal $\mathrm{TEM}_{00}$ mode with quasi-perfect Gaussian shape. The thermo-electrically stabilized VECSEL is pumped by a $1.55 \mu \mathrm{m}$ laser diode with $10 \mathrm{~W}$ optical power and $5 \mathrm{~ns}$ long pulses at a repetition rate of $100 \mathrm{kHz}$. The output peakpower of the mid-IR radiation at room temperature is a few $\mathrm{mW}$. A scan frequency of $500 \mathrm{~Hz}$ was selected as an optimum between scanningspeed and the number of spectral data points within the scan.

The VECSEL has spectroscopically attractive properties, such as diffraction limited $\left(\mathrm{M}^{2}\right.$ $=1.14$ ), single mode beam emission with very narrow beam divergence $\left(<1.7^{\circ}\right)$, and continuous, mode-hop free tuning up to $6 \%$ around the center wavelength. However, being still a relatively new laser source, mid-IR VECSEL exhibits some inherent issues that need to be addressed as discussed below. Perhaps most demanding is the achievement of a stable and constant signal over the entire tuning range, given the considerable pulse-to-pulse power variations and the wavelength dependency of the output power (see Figure 2). This latter is a modulation caused by the variation of the intrinsic gain profile and possible interference effects within the VECSEL structure, which considerably constrains the usable spectral coverage range. To compensate for pulse-to-pulse amplitude variations, we employed the technique of pulse normalization using a reference optical path with temporal gating as proposed by Nel- son et al. ${ }^{29}$ This method relies on the large optical path difference between the sample pulse passing trough a multipass cell and the reference pulse directly reaching the detector, i.e. on a time delay that enables to temporally resolve and normalize the two pulses using a single detector. For this approach to work efficiently it is, however, mandatory that the light maintains a fixed state of polarization. This is due to polarization sensitive elements such as beamsplitter and dielectric coated mirrors of the multipass cell. The polarization direction of the light emitted by the VECSEL, however, randomly fluctuates between two preferential polarization directions orthogonal to each other. This considerably diminishes the effectiveness of the pulse normalisation method by inducing additional intensity noise on the reference and sample paths that cannot be compensated for. Therefore, a polarizer was placed into the laser beam to select a single polarization angle. The best compromise for maintaining a good signalto-noise ratio was to rotate the main polarizer axis by $45^{\circ}$ from the two orthogonal planes, a configuration at which the polarizer is partly transparent for every pulse.

Another limiting factor is the etaloning effect of the VECSEL's cavity that is seen as a sinusoidal modulation of the laser intensity across the entire scanning range (see also Figure 2). This can efficiently be suppressed by background subtraction using the transmittance of the multipass cell filled with dry $\mathrm{N}_{2}$ at pressure similar to that of the sample gas. For best results, a stable laser operation is required, i.e. no drift in the emission frequency of the VECSEL. Therefore, a single-pass $(14 \mathrm{~cm})$ reference cell filled with $4 \% \mathrm{CH}_{4}$ has been added, and the position of the absorption lines are used as frequency reference. A feedback signal is applied to the piezo-voltage to correct the cavity position and maintain the VECSEL emission frequency within $0.025 \mathrm{~cm}^{-1}$.

Finally, due to the pulsed operation, the VECSEL emission line width is broadened $(\approx$ $1 \mathrm{~cm}^{-1}$ ) by thermal chirp effect. This implies a considerable effort on the spectral analysis process, considering the fact that the laser emission line profile has an asymmetric tailing and it also 


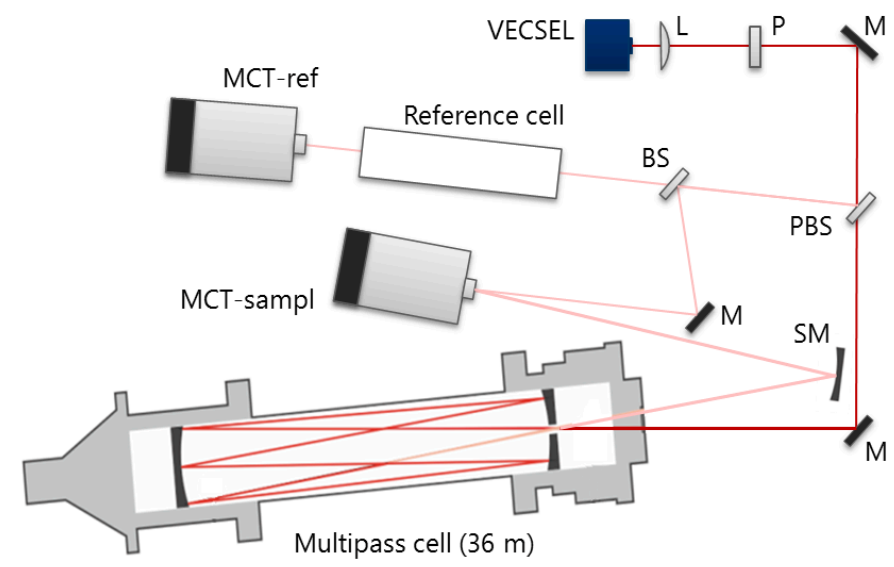

Figure 3: Schematic of the optical setup. L: lens; P: polarizer; M: plane mirror; PBS and BS: (pellicle) beamsplitter; MCT: mercurycadmium-telluride detector for sample (sampl) and reference (ref) path; SM: sperical mirror.

changes while tuning across the gain curve.

The final layout of the optical setup implementing all the requirements discussed above is schematically shown on Figure 3. The laser pulse first passes through an AR-coated planoconvex $\mathrm{CaF}_{2}$ lens followed by a linear polarizer and is then split into reference and sample pulse using a coated pellicle beamsplitter. The reference pulse goes through another beamsplitter and its reflected fraction is focused onto a thermoelectrically cooled IR detector (PVI-3TE-4, Vigo SA, Poland). This serves as intensity normalization for the sample pulse that goes through the astigmatic mirror Herriott multipass cell (AMAC-36, Aerodyne Research, Inc., USA) and reaches the same detector with a time delay of $120 \mathrm{~ns}$. The transmitted fraction of the reference pulse is passing through the single-pass cell and then arrives at a second IR detector. $\mathrm{A} \mathrm{CaF}_{2}$ window of $2 \mathrm{~mm}$ thickness can be placed into the reference path to generate etalon fringes with free spectral range of $1.77 \mathrm{~cm}^{-1}$, which are then used to determine the frequency scale.

To capture the time-resolved profiles of the optical pulses, a fast IR-detector with $50 \mathrm{MHz}$ bandwidth preamplifier (MIPAC-F-50, Vigo $\mathrm{SA}$, Poland) is combined with a high-speed digitizer (PCI-5124, National Instruments, USA). The laser pulser $(100 \mathrm{kHz})$ and piezo-tuning $(500 \mathrm{~Hz})$ is controlled by a multipurpose data acquisition board (DAQ, PCI-6111, National Instruments, USA). The devices are synchronized through the real-time system integration (RTSI) bus interface, using the internal reference clock of the digitizer. Data acquisition is performed at $200 \mathrm{MS} / \mathrm{s}$ at 12-bit resolution on two channels. The digitizer is triggered by the laser trigger signal and acquires data for $250 \mathrm{~ns}$. This time-window is wide enough to cover both the reference and sample optical pulses arriving at the detector. A complete spectral sweep is assembled from 200 individual laser pulses. A total of 250 consecutive scans are bundled in the on-board memory of the digitizer and then sent to the computer for further data processing. The correct allocation and book-keeping of the individual data segments is based on an additional gate signal of similar length as the ramp signal for the piezo-tuning, synchronously generated by the DAQ.

\section{Spectral analysis}

A custom-written LabVIEW-based program controls the hardware and processes the data in real-time, i.e. co-aligns the consecutive pulses within a spectral sweep, performs the pulse normalization, co-averages individual scans, and displays the scaled absorption spectrum. A separate module of the program performs parallel real-time fitting of both the sample- and reference-cell absorption spectra, respectively. The latter is used to monitor the frequency drift of the laser emission by fitting a $\mathrm{CH}_{4}$ absorption line and apply the necessary feedback to the piezo-tuning voltage as previously discussed.

The broad and asymmetric emission line width of the VECSEL strongly influences the absorption features and hinders the use of an absorbance model based on either the PNNL or the HITRAN database for accurate spectral analysis. Therefore, absorption spectra for each individual compound were recorded using certified calibration gases (see next section for more details). The recorded spectra were then smoothed with a Savitzky-Golay filter to remove high-frequency spectral noise, and converted to absorbance values as used by the PNNL database, i.e. in $\mathrm{ppm}^{-1} \mathrm{~m}^{-1}$ units. 

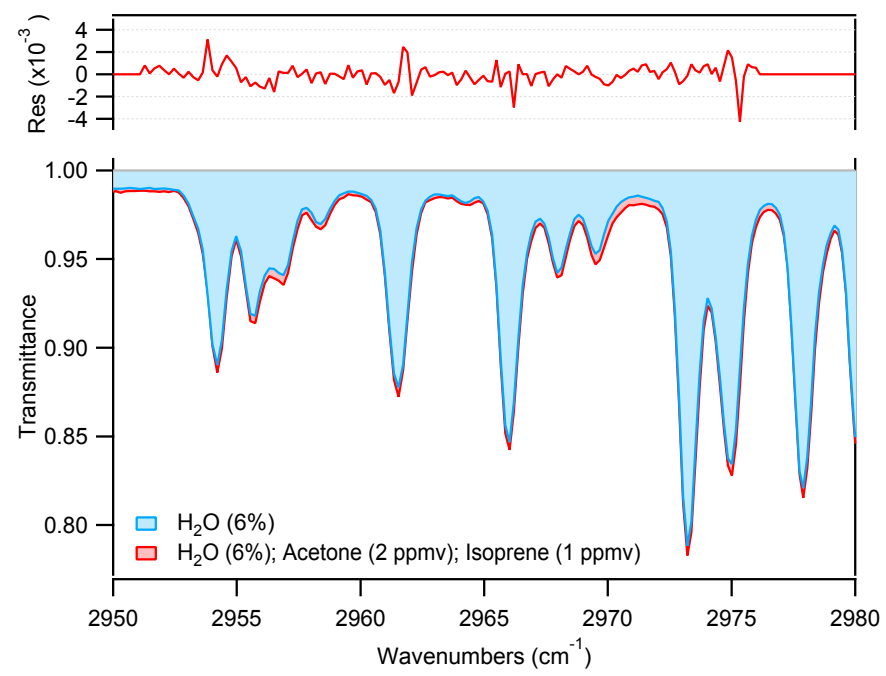

Figure 4: Fitted absorption spectrum of a humid gas mixture containing acetone and isoprene at mixing ratio of 2 and 1 ppmv, respectively and water vapor at $6 \%$ (red trace), and as reference, the absorption spectrum of the water vapor only at the same amount (blue trace). The top panel indicates the residual of the fit to the measured data.

These templates are taken as reference spectra to create a model of the absorbance of the gasmatrix.

The measured transmission spectrum of the breath-like mixtures is well modeled by the sum of the individual absorbance templates of the different molecular species. For water, however, the situation is significantly different: some of the water lines in the selected spectral region are saturated at high humidity levels $(\geq 6 \%)$. This leads, in combination with the substantial broadening due to the large laser emission line width, to a significant concentration dependence of the water spectral shapes. To account for this effect, individual water templates were recorded within the expected experimental humidity range $(0-8 \%)$ in $0.5 \%$ humidity steps. These templates were grouped into an array and fed into the fit function. In each iteration step, the fit function calculates from the array an interpolated template to account for the contribution of the water to the measured transmission spectrum. The fitting function uses the Levenberg-Marquardt algorithm to determine the concentration of each compound. This ap- proach was proven to be a robust method allowing the rapid and accurate computation of the gas concentrations under a wide variety of conditions. An example of fitted data is shown in Figure 4, where acetone and isoprene at mixing ratios of 2 and 1 ppmv, respectively is quantified in the presence of $6 \%$ water vapor.

\section{Calibration and Validation}

Three gas cylinders of certified calibration gases (Messer, Switzerland) were used, each containing an individual compound (acetone, isoprene, or methane) in dry nitrogen at a mixing ratio level of $20 \pm 1$ ppmv. Mass flow controllers (MFC 2112, Axetris AG, Switzerland) maintained a constant gas flow through the multipass cell and a precision needle-valve (Swagelok, USA) placed at the downstream side was used to adjust the pressure level to 300 mbar in the cell.

In order to quantitatively investigate the influence of changing water vapour on acetone measurements, we deployed a calibration gas generator (HovaCAL, IAS GmbH, Germany) that can produce and continuously deliver precise gas vapor mixtures with selectable humidity. The device consists of an evaporator and a supply unit that controls and regulates the flow of water, carrier gas, and the temperature of the evaporator. A peristaltic pump continuously delivers the liquid to the evaporator, while an electronic balance (AE200, MettlerToledo GmbH, Switzerland) monitors the consumption. The generated gas vapor mixture is exactly defined by the amount of water evaporated in relation to the gas flow in the water evaporator. Applying this method, we generated gas mixtures with water vapor content up to $8 \%$. To prevent water condensation in the gas sampling line, the gas pressure was reduced to 300 mbar by a precision needle valve placed directly after the evaporator heated to $180^{\circ} \mathrm{C}$.

A further benefit of this method is the possibility to gravimetrically prepare complex humid mixtures by adding well defined amounts of acetone, isoprene, and alcohols to the water reservoir. This enables a flexible and rapid change of compounds that can be used for a quantitative investigation of the spectral inter- 
ferences between various species and a reliable calibration with large variety of humid mixtures in the lower ppmv range, limited only by the mass-resolution $(0.1 \mathrm{mg})$ of the deployed balance. The evaporation of volatile compounds from the liquid mixture is avoided by applying a thin film of paraffin oil (Sigma-Aldrich) using a syringe. Due to its viscosity, the oil droplets create a uniform layer that hermetically seals the fluid surface. ${ }^{30}$ Without this vapor barrier layer, the acetone concentration in the liquid mixture dropped exponentially with a time constant of about ten hours. The gas handling system also allows for controlled mixing of the certified calibration gases from the gas cylinders with the humidified $\mathrm{N}_{2}$ gas stream. The gas transfer system was fully based on $1 / 8$ " diameter stainless steel, which was treated with Sulfinert ${ }^{\circledR}$ to reduce wall losses and memory effects.

\section{Results and discussion}

Examples of absorption spectra recorded using various gas-mixtures are shown in Figure 5. The overlayed spectra also illustrate the additive character of the individual absorbances of a gas mixture. Moreover, Figure 5 clearly shows that despite the overlap between isoprene, acetone, and methane absorption, the species are easily discernable allowing for unambiguous and quantitative determination of their concentrations as has been verified experimentally. In addition, potential interferences and cross-sensitivities have been determined as shown below.

First, the instrument response was investigated by stepwise addition of the certified calibration gas containing 20 ppmv acetone mixed in dry $\mathrm{N}_{2}$ to the humidified gas stream $\left(\mathrm{N}_{2}\right.$ with $4 \%$ water vapor). The results are summarized in Figure 6 and indicate an excellent linear behavior of the spectrometer within a concentration range that is expected for human breath samples - the higher values being more typical only for subjects with acute diabetes.

The major objective of this study is to obtain accurate and high precision acetone measure-

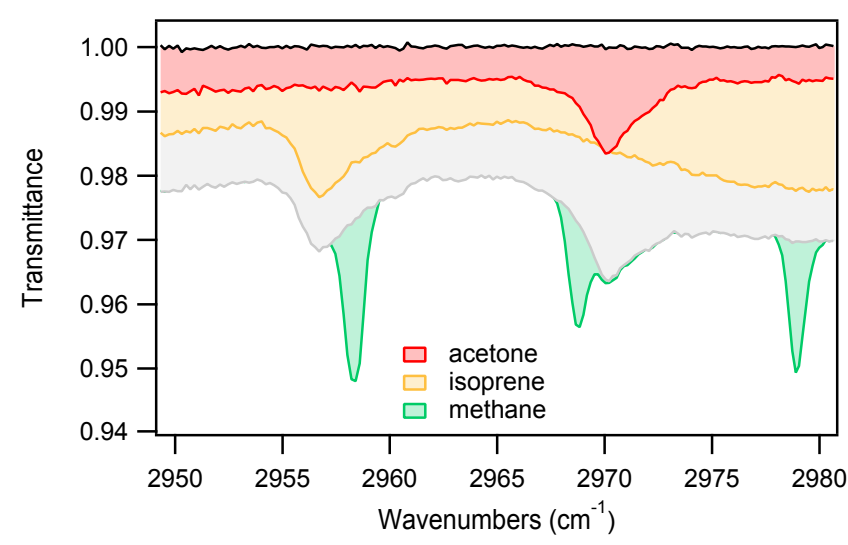

Figure 5: Measured absorption spectra of pure acetone (red) and isoprene (yellow) diluted in dry $\mathrm{N}_{2}$ to 5 ppmv and 6.5 ppmv, respectively, followed by a mixture of the two compounds (grey), and the addition of 3 ppmv methane (green).

ments even in the case of samples with water content of up to $6 \%$, corresponding to saturated water pressure at $37^{\circ} \mathrm{C}$, i.e. at body temperature. This would correspond to direct measurements of human breath without sample preparation.

In order to determine selectivity, acetone calibration gas is mixed 1:10 with $\mathrm{N}_{2}$ and increasing water vapor. The spectra are analyzed using the aforementioned empirical templates for acetone, isoprene, and water. The results are summarized in the Figure 7 . The observed dependency of both compounds on water vapor concentration is less than 1 ppmv for the full range of water vapor $(0-7 \%)$ and it can be modelled with polynomial functions of $3^{r d}$ and $4^{\text {th }}$ order for acetone and isoprene, respectively. Repeated measurements indicate that these dependencies are robust and reproducible.

Furthermore, the range of water vapor is well constrained by typical humidity in breath: the samples are either analyzed directly (saturated water vapor pressure at $37^{\circ} \mathrm{C}$ ) or in bags (saturated vapor pressure at room temperature, e.g. about $23^{\circ} \mathrm{C}$ ). Considering these two cases, it is obvious that in the former case the measured acetone values need almost no corrections as the dependency is weaker than the measurement precision, while in the case of samples with higher humidity, a linear correction would 


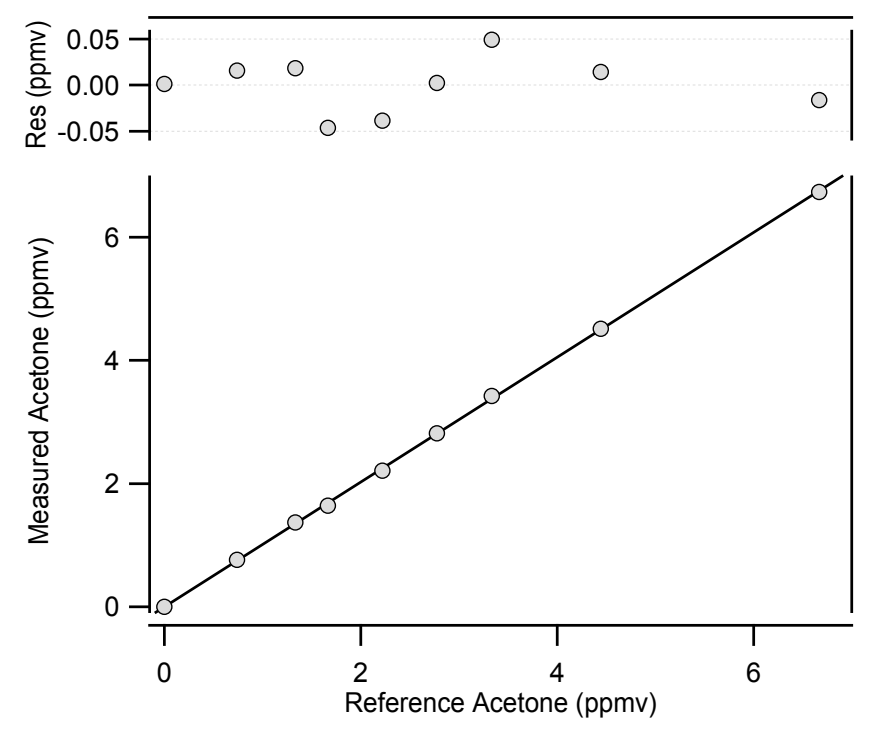

Figure 6: Instrumental response to changing acetone concentration in the presence of $4 \%$ water vapor. A mass flow controller was employed to induce a stepwise dilution of the calibration gas containing 20 ppmv acetone in $\mathrm{N}_{2}$.

be enough.

Similarly, the effect of changing methane concentration on acetone measurements was studied. For this purpose, a gas matrix containing 2.4 ppmv acetone, 1 ppmv isoprene, and $4 \%$ water has been generated, whereas the $\mathrm{CH}_{4}$ mixing ratio was repeatedly varied between 0 and 6 ppmv. No change in the acetone and isoprene values within the analytical precision was observed. Likewise, varying the isoprene mixing ratio from 0 to 10 ppmv had no detectable influence on the measured acetone concentration values.

The potentially important interferences from alcohol species was also considered. To investigate their influence, $50 \mu \mathrm{L}$ acetone was diluted in $90 \mathrm{~mL}$ water and used as liquid supply for the HovaCAL. The generated gas mixture contained 4 ppmv acetone at $4 \%$ water vapour and was continuously flown through the multipass absorption cell. The liquid supply was then increasingly spiked with $20 \mu \mathrm{L}$ ethyl-alcohol in five consecutive steps, leading to an ethyl-alcohol concentration increase in the gas mixture up to $35 \mathrm{ppmv}$, corresponding to about $0.01 \%$ blood alcohol content. The same procedure was repeated for methyl-

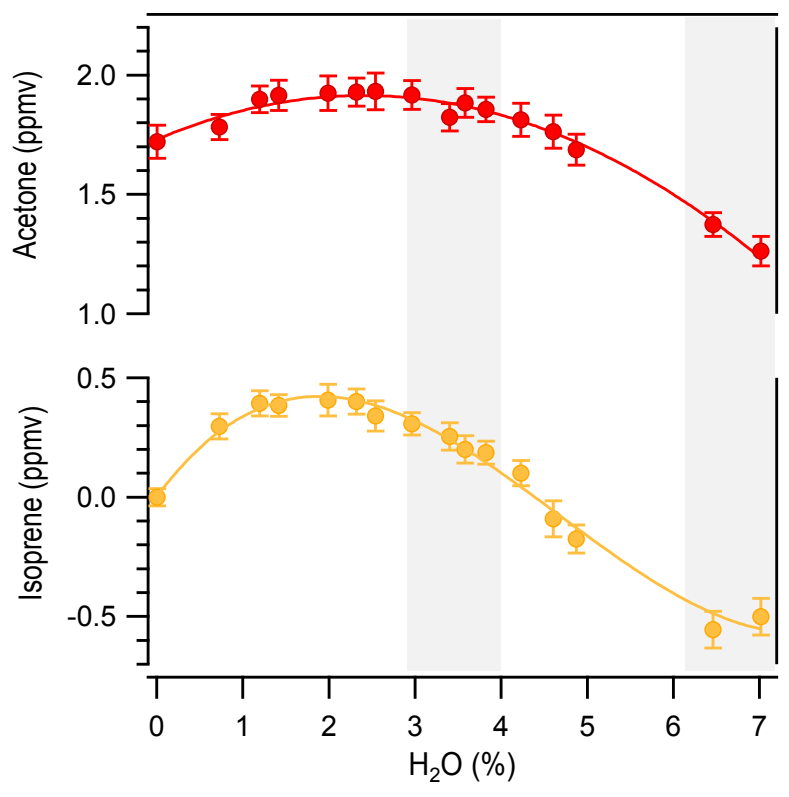

Figure 7: The effect of spectral interference due to water on the retrieved acetone and isoprene mixing ratio values. Gray shaded areas indicate the range of water vapor expected either from bags (sampled and stored at room temperature) or direct breath samples measured at temperatures equivalent or higher then the human body.

alcohol. We found just marginal dependence $(8 \pm 0.5 \mathrm{ppbv} / \mathrm{ppmv})$ of acetone on both alcohol species. However, we verified also the situation when the alcohol species were not included in the fitting model. In this case, a 40 times larger dependency of acetone on alcohol content is observed, which can then have dramatic consequences on the diagnostic results. ${ }^{31}$ Therefore, we propose the multi-compound fitting approach instead of a simple ethanol correction. As the covered spectral range in our case is broad enough to deliver sufficient confinement to the fit, a robust, reliable, and accurate determination of individual species from a gas-mixture is guaranteed even for situations of varying concentration of the various compounds.

Finally, a fitting model with six individual species (see Table 1) was set up and the absorption signal generated by a dry, breath-like gas mixture (acetone, isoprene and methane diluted with $\mathrm{N}_{2}$ ) was continuously analyzed at one second time resolution over two hours. This blank 


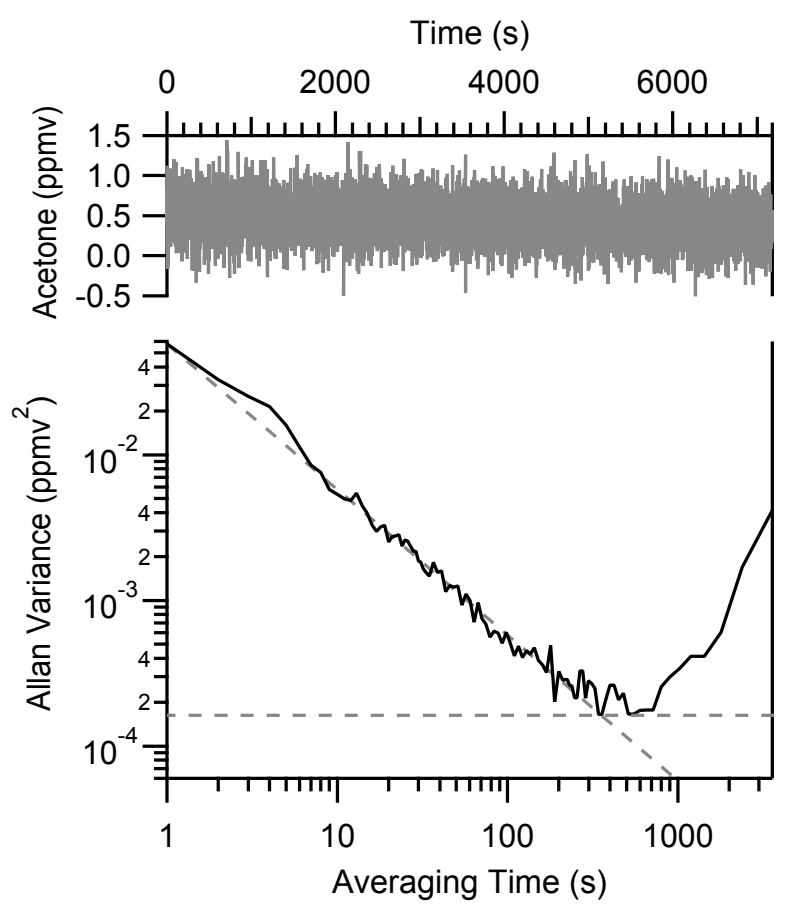

Figure 8: Time series and associated Allan variance plot of acetone.

sample measurement is used to determine the precision, given as the Allan variance, ${ }^{32}$ under optimal (dry) conditions and to evaluate the long-term performance of the instrument. As shown in Figure 8, the measurement is dominated by white-noise, which is mainly given by the detector dark-noise due to low laser output power, but also by the random laser fluctuations as discussed above. The root mean square noise level at $1 \mathrm{~s}$ is about $240 \mathrm{ppbv}$, and from the associated Allan variance plot an optimum averaging time of 5 min can be derived, leading to a relative precision of $13 \mathrm{ppbv}$ or to an absorbtion noise level of $2.3 \times 10^{-5}$. Considering the $36 \mathrm{~m}$ optical path this would be equivalent to an absorbance per unit path length noise of $6.4 \times 10^{-9} \mathrm{~cm}^{-1}$. This is similar or about an order of magnitude better than reported works adopting laser absorption techniques. ${ }^{24,28,33,34}$

Following these characterization steps, the spectroscopic instrument was deployed to sequentially measure dry calibration gas, a sample of alveolar breath of a person, and ambient air, respectively. In all cases, the sample gas was flowing continuously through the multipass cell at a rate of $50 \mathrm{~mL} \mathrm{~min}^{-1}$. Figure 9 shows the results for the three most abundant com-
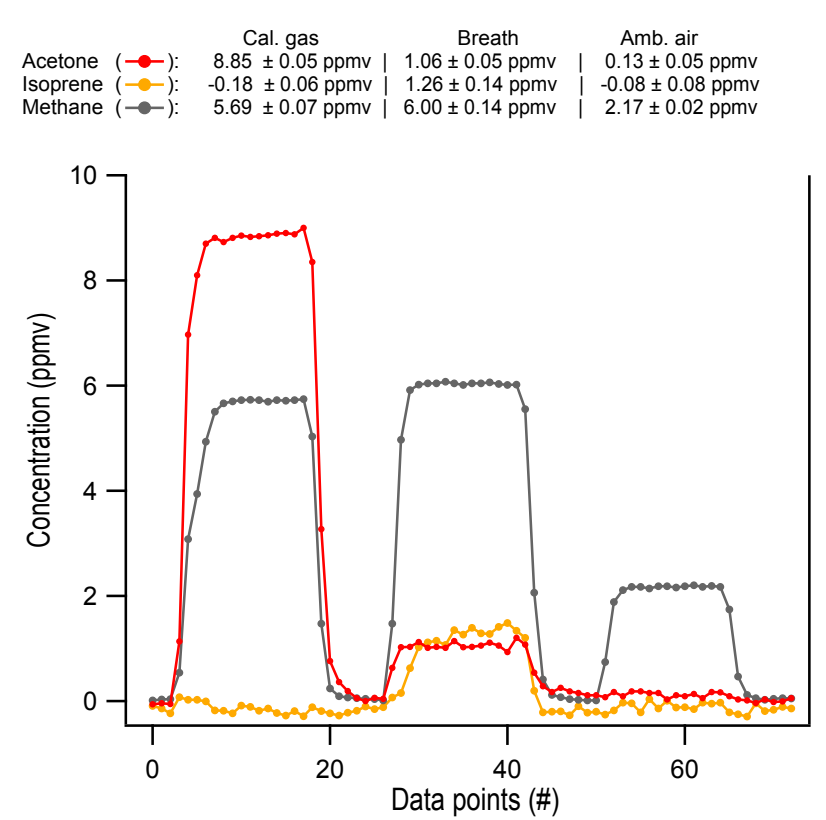

Figure 9: Analysis of calibration gas, a human breath sample collected in a Tedlar bag and ambient air. The measured concentrations $( \pm 1 \sigma$ standard deviation) of the compounds are given at the top.

pounds of such a sequence of measurements. The instrument is currently prepared for a pilot clinical study that aims to explore correlations between breath acetone variations and lifestyle interventions, such as energy deficient diet and physical exercises, targeted to stimulate fat oxidation.

Table 1: Summary of analytical performance of the VECSEL based spectrometer given as limit of detection (LOD in ppbv) at 1s and after 5 min averaging time which corresponds to the Allan variance minimum.

\begin{tabular}{lrr}
\hline \hline \multirow{2}{*}{ Compound } & \multicolumn{2}{c}{ LOD $(1 \sigma)$} \\
\cline { 2 - 3 } & $1 \mathrm{~s}$ & $5 \mathrm{~min}$ \\
\hline Acetone & 241 & 13 \\
Isoprene & 152 & 6.5 \\
Methane & 63 & 3 \\
Ethanol & 257 & 40 \\
Methanol & 274 & 13 \\
\hline
\end{tabular}




\section{Conclusions}

A laser spectroscopic approach for the quantitative and real-time analysis of acetone and isoprene in exhaled human breath has been described. The wide spectral coverage allows for simultaneous measurement of the most important interfering species such as alcohols, methane, and water. This gives us the unique opportunity to verify and fully characterize the response of the spectrometer across a wide range of sample conditions that may be experienced in human breath. The results confirm the capability of the instrument to deliver reliable acetone concentration values in complex gas mixtures even without any sample pretreatment, i.e. without a water removal or selective pre-concentration step. Therefore, the VECSEL based system provides a promising noninvasive tool for on-line monitoring of VOCs in human breath.

Acknowledgement This work was supported by the NRP "Healthy Nutrition and Sustainable Food Production" funding agency (SNF) under the project name "Preventing obesity". The authors thank Ivo Strebel for the detailed ray-tracing investigations and Yannick Stöferle for assistance in performing validation measurements of the VECSEL-spectrometer.

\section{References}

(1) W., M.; Schubert, J.; NoeldgeSchomburg, G. Clin. Chim. Acta 2004, 347, 25-39.

(2) Risby, T.; Solga, S. Appl. Phys. B: Lasers Opt. 2006, 85, 421-426.

(3) Boots, A.; van Berkel, J.; Dallinga, J.; Smolinska, A.; Wouters, E.; van Schooten, F. J. Breath Res. 2012, 6, 027108-027129.

(4) Amann, A., Smith, D., Eds. Breath Analysis for Clinical Diagnosis and Therapeutic Monitoring; World Scientific, 2012.
(5) Mochalski, P.; King, J.; Klieber, M.; Unterkofler, K.; Hinterhuber, H.; Baumann, M.; Amann, A. Analyst 2013, 138, $2134-2145$.

(6) Lindinger, W.; Hansel, A.; Jordan, A. Chem. Soc. Rev. 1998, 27, 347-354.

(7) Smith, D.; Španěl, P. Mass Spectrom. Rev. 2005, 24, 661-700.

(8) King, J.; Kupferthaler, A.; Unterkofler, K.; Koc, H.; Teschl, S.; Teschl, G.; Miekisch, W.; Schubert, J.; Hinterhuber, H.; Amann, A. J. Breath Res. 2009, 3, 027006.

(9) Schubert, R.; Schwoebel, H.; MauMoeller, A.; Behrens, M.; Fuchs, P.; Sklorz, M.; Schubert, J.; Bruhn, S.; Miekisch, W. Metabolomics 2012, 8, 1069-1080.

(10) Samudrala, D.; Lammers, G.; Mandon, J.; Blanchet, L.; Schreuder, T.; Hopman, M.; Harren, F.; Tappy, L.; Cristescu, S. Obesity 2014, 22, 980-983.

(11) Stone, B.; Besse, T.; Duane, W.; Dean Evans, C.; DeMaster, E. Lipids 1993, 28, 705-708.

(12) Risby, T. H.; Tittel, F. K. Opt. Eng. 2010, 49, 111123-14.

(13) Faist, J.; Gmachl, C.; Capasso, F.; Sirtori, C.; Sivco, D.; Baillargeon, J.; Cho, A. Appl. Phys. Lett. 1997, 70, 2670-2672.

(14) Meyer, J.; Vurgaftman, I.; Yang, R.; RamMohan, L. Electron. Lett. 1996, 32, 4546.

(15) Hugi, A.; Maulini, R.; Faist, J. Semicond. Sci. Technol. 2010, 25, 083001.

(16) Riedi, S.; Hugi, A.; Bismuto, A.; Beck, M.; Faist, J. Appl. Phys. Lett. 2013, 103, 031108.

(17) Fill, M.; Khiar, A.; Rahim, M.; Felder, F.; Zogg, H. J. Appl. Phys. 2011, 109. 
(18) Rahim, M.; Khiar, A.; Fill, M.; Felder, F.; Zogg, H. Electron. Lett. 2011, 47, 10371039 .

(19) Sharpe, S.; Johnson, T.; Sams, R.; Chu, P.; Rhoderick, G.; Johnson, P. Appl. Spectrosc. 2004, 58, 1452-1461.

(20) Rothman, L. et al. J. Quant. Spectrosc. Radiat. Transfer 2013, 130, 4-50.

(21) Turner, C.; Spanel, P.; Smith, D. Physiol. Meas. 2006, 27, 321-337.

(22) Španěl, P.; Dryahina, K.; Rejšková, A.; Chippendale, T. W.; Smith, D. Physiol. Meas. 2011, 32, N23-31.

(23) King, J.; Kupferthaler, A.; Frauscher, B.; Hackner, H.; Unterkofler, K.; Teschl, G.; Hinterhuber, H.; Amann, A.; Högl, B. Physiol. Meas. 2012, 33, 413-428.

(24) Ciaffoni, L.; Hancock, G.; Harrison, J.; Van Helden, J.; Langley, C.; Peverall, R.; Ritchie, G.; Wood, S. Anal. Chem. 2013, 85, 846-850.

(25) Basum, G. V.; Halmer, D.; Hering, P.; Mürtz, M. Breath Analysis for Clinical Diagnosis and Therapeutic Monitoring; World Scientific, 2012; Chapter 5, pp 6774 .

(26) Hancock, G.; Langley, C.; Peverall, R.; Ritchie, G.; Taylor, D. Anal. Chem. 2014, 86, 5838-5843.

(27) Blaikie, T. J.; Couper, J.; Hancock, G.; Hurst, P.; Peverall, R.; Richmond, G.; Ritchie, G.; Taylor, D.; Valentine, K. Anal. Chem. 2016, (just accepted manuscript).

(28) Arslanov, D. D.; Swinkels, K.; Cristescu, S. M.; Harren, F. J. M. Opt. Express 2011, 19, 24078-24089.

(29) Nelson, D. D.; Shorter, J.; McManus, J. B.; Zahniser, M. S. Appl. Phys. B: Lasers Opt. 2002, 75, 343-350.
(30) Iannone, E. Labs on Chip: Principles, Design and Technology; CRC Press, Taylor \& Francis Group: Boca Raton, FL, 2015.

(31) Centeno, R.; Mandon, J.; Harren, F. J. M.; Cristescu, S. M. Photonics 2016, 3, 22.

(32) Werle, P.; Mücke, R.; Slemr, F. Appl. Phys. B: Photophys. Laser Chem. 1993, 57, 131-139.

(33) Wang, C.; Mbi, A. Meas. Sci. Technol. 2007, 18, 2731.

(34) Sun MX, G. Z.; Gong, Z.; Sun, M.; Jiang, C.; Wang, Z.; Kang, M.; Li, Y.; Wang, C. J. Anal. Bioanal. Tech. 2014, S7, 1-8. 